\title{
PREFACE
}

\section{Microplastic and Nanoplastic Pollution: Characterization, Transport, Fate, and Remediation Strategies}

(C) Higher Education Press 2022

Plastics continues to have a critical and essential role in human society such as food packing, product packages and building materials. In the meantime, it has been recognized as a global sustainability priority to study and mitigate pollution of plastics and the associated unknown impacts from ultrafine plastic particles. Microplastic $(<5 \mathrm{~mm})$ particles, for instance, have been detected in the aquatic environment globally and have raised scientific interests and environmental concerns. Microplastics (MPs) can enter rivers through a variety of pathways (e.g., wastewater effluent, breakdown of plastic debris) and can negatively impact aquatic organisms through both direct injection with food and indirect contamination from absorbed toxins. MPs can absorb heavy metals, pathogens, and organic contaminants, mainly persistent organic pollutants (POPs) that can be ingested by organisms and introduced into the food web. Meanwhile, MPs may also release potentially toxic substances (e.g., plasticizers, flame retardants and antimicrobial agents) during weathering and degradation. An increasing number of papers are being published during the last years related to the effects of MPs as well as with synergistic effects when associated with MPs and their byproducts such as nanometer sized plastics or nanoplastics.

This special issue successfully garnered a collection of research articles that addressed various topics of microplastic or nanoplastic pollution, characteristic, and environmental fate in the water environment. Particularly, this issue reported the seasonable occurrence and distribution of microplastics in different regions (e.g., China and India) from high-latitude nature reserve to sediments and coastal marine environments. Moreover, new sampling and analytical methodologies (e.g., fluorescent labeling) to identify MPs in diver matrixes (e.g., wastewater, sediment, and biota) are critically reviewed. The information is critical for scientists, business leaders, legislators, and the public are to explore and develop solutions that lessen society's plastic footprint.

We thank all authors, reviewers, and editorial staff of FESE for their efforts and contributions to this special issue.

Guest Editors:

Wen Zhang

Department of Civil and Environmental Engineering, New Jersey Institute of Technology (wen.zhang@njit.edu)

Melissa Pasquinelli

College of Natural Resources, North Carolina State University (melissa_pasquinelli@ncsu.edu)

Yang Li

School of the Environment

Beijing Normal University (liyang_bnu@bnu.edu.cn)

Received December 7, 2021

Special Issue-Microplastic and Nanoplastic Pollution: Characterization, Transport, Fate, and Remediation Strategies (Responsible Editors: Wen Zhang, Melissa Pasquinelli \& Yang Li) 